\title{
Development of the radio astronomical method of cosmic particle detection for extremely high-energy cosmic ray physics and neutrino astronomy
}

\author{
Igor Zheleznykh ${ }^{1,2, a}$, Rustam Dagkesamanskii ${ }^{3}$, Leonid Dedenko ${ }^{4}$, and Grigorii Dedenko ${ }^{5}$ \\ 1 Institute for Nuclear Research, Russian Academy of Sciences, Russia \\ 2 Moscow Institute of Physics and Technology, Russia \\ 3 Lebedev Physics Institute, Russian Academy of Sciences, Pushchino Radio Astronomy Observatory, Russia \\ ${ }^{4}$ Moscow State University, Department of Physics, Russia \\ 5 Nuclear Research National University “Moscow Engineering Physics Institute”, Russia
}

\begin{abstract}
The proposal to use ground based radio telescopes for detection of Askaryan radio pulses from particle cascades arising when extremely high-energy (EHE $>10^{20} \mathrm{eV}$ ) cosmic rays (including neutrinos) interact with the lunar regolith of multi gigaton mass was made at the end of 1980s in the framework of the Russian (Soviet) DUMAND Program. During more than a quarter of century a number of lunar experiments were carried out mainly in the $1-3 \mathrm{GHz}$ frequency range using the large radio telescopes of Australia, USA, Russia and other countries but these experiments only put upper limits to the EHE cosmic rays fluxes. For this reason, it would be of great interest to search for nanosecond radio pulses from the Moon in a wider interval of frequencies (including lower ones of $100-350 \mathrm{MHz}$ ) with larger radio detectors - for example the giant radio telescope SKA (Square Kilometer Array) which is constructed in Australia, New Zealand and South Africa. In this paper possibilities are discussed to use one of the most sensitive meter-wavelength $(\sim 110 \mathrm{MHz})$ Large Phased Array (LPA) of $187 \times 384 \mathrm{~m}^{2}$ and the wide field of view meter-wavelength array of the Pushchino Radio Astronomy Observatory as prototypes of low frequency radio detectors for lunar experiments. The new scheme for fast simulation of ultrahigh and extremely high-energy cascades in dense media is also suggested. This scheme will be used later for calculations of radio emission of cascades in the lunar regolith with energies up to $10^{20} \mathrm{eV}$ and higher in the wide frequency band of $0.1-\mathrm{a}$ few $\mathrm{GHz}$.
\end{abstract}

\section{Introduction: DUMAND and a new generation of neutrino telescopes}

In the late fifties - early sixties it was proposed that a number of fundamental problems of Particle Physics and Astrophysics (Astronomy) could be studied using underground (underwater) installations for detecting high energy (HE) neutrinos produced in the Earth's atmosphere and in space [1-3]. The first HE atmospheric neutrinos were detected in 1965 with the help of scintillation detectors of tens $\mathrm{m}^{2}$ located in deep gold mines in India (A. Wolfendale, M. Menon, S. Miyake, et al.) and in South Africa (F. Reines, W. Kropp, H. Sobel, et al.).

In 1966, F. Reines and J. Sellschop suggested to build COLOSSUS - a large deep underground detector of $10^{5}$ tons to register neutrinos from the atmosphere and also beyond [4]. In 1973-1976 the idea to construct the large underwater optical neutrino telescope DUMAND of cubic kilometer size had been developed by F. Reines, J. Learned, H. Blood, H. Bradner, A. Roberts, G. Wilkins, et al. (see [5]).

The hydro-acoustical detection of electron-hadron cascades of ultrahigh-energies (UHE $>10^{15} \mathrm{eV}$ ) and extremely high-energies was also considered in

\footnotetext{
a e-mail: zhelezny@minus.inr .ac.ru
}

1976-1979 (G. Askaryan and B. Dologoshein, T. Bowen, J. Learned).

The great value of the DUMAND program 1980-1995 (in particular the DUMAND Workshop in Hawaii in 1976) for developing deep underwater neutrino telescopes in the Ocean near Hawaii, in Baikal, in the Mediterranean Sea but also for the development and construction of the large neutrino detector IceCube in Antarctica and smaller underground water-filled detectors such as IMB and Kamiokande was emphasized by J. Learned and Ch. Spiering in their recent article [6].

It is significant that the creation of the deep underground water Cherenkov detectors of $5 \times 10^{3} \mathrm{~m}^{3}$ and more had become a real compromise between COLOSSUS and DUMAND and that a first step to developing a huge Antarctic under-ice optical neutrino telescope IceCube had been made by F. Halzen and J. Learned in 1988 [7].

In Sect. 2 we should like to pick out specific features of the Russian (Soviet) DUMAND program led by M.A. Markov in 1980-1994, in particular the development of new branches of DUMAND:

- the Radio Antarctic Muon and Neutrino Detection (RAMAND) to search for astrophysical sources of ultrahigh-energy (UHE $>10^{14} \mathrm{eV}$ )neutrinos using the Antarctic ice as a neutrino target; 
- the Radio Astronomical Method of the Hadron and Neutrino Detection project (RAMHAND) to study EHE cosmic rays bombarding the Moon.

In Sect. 3 the results of the first RAMHAND-type experiments are considered and great prospects of possible experiments with larger radio arrays SKA, LOFAR, LPA (Pushchino) are discussed. The new scheme for fast simulation of ultrahigh-energy and extremely high-energy cascades in dense media is suggested (see Sect. 4).

\section{The Russian (Soviet) DUMAND and development of large-scale Radio Neutrino Telescopes}

After the Workshops DUMAND-78 at La-Jolla and DUMAND-79 (August 1979) at Khabarovsk and Lake Baikal, Fred Reines and Moisey Markov agreed (in October 1979) that the USSR would join the International DUMAND Hawaii Project. But such a collaboration was not approved by the US authorities (see more about that in [5]).

In 1980 Markov headed the Soviet DUMAND program which included more than 10 Institutes from Kaliningrad (former Konigsberg) up to Vladivostok. The goal of the Soviet DUMAND project was the development of the deep underwater optical and acoustical methods of UHE and EHE neutrino detection in the World Ocean (in particular in the Mediterranean Sea) and in Lake Baikal. And from the beginning there were discussed alternative methods of UHE and EHE neutrino detection.

One of arguments to support the construction of largerscale (multi gigatons) detectors was of basic interest to study the upper boundary of the neutrino energy spectrum and spectra of CR particles. The existence of neutrinos with energies higher than $10^{20} \mathrm{eV}$ might be related to the fundamental problem of the Physics of Elementary Particles - the possible existence of super-heavy particles. It was emphasized that UHE and EHE neutrinos may arise not only in secondary processes of decays of pions, kaons, charmed particles produced in the interactions of UHE and EHE protons and nuclei with the pulsar shells, interstellar gas, relic photons, etc. They may result from decays and interactions of super-heavy particles. Some of the first "top-bottom" models of the EHE particle (super GZK neutrino) production, considered in 1979-1981, were as following: the decays of Mini-Black Holes [8], interactions (decays) of such hypothetical super heavy particles as magnetic monopoles, GUT particles etc. [9].

The first proposal to detect radio emission of electronphoton cascades, induced in the crust of the Earth by EHE neutrinos from the lower hemisphere and continuing to propagate in the atmosphere in a direction close to horizontal, was made in 1981 [9] (see also [10]).

Askaryan's idea of the 1960s [11] to detect coherent Cherenkov radio emission of cascades produced by cosmic rays in dense dielectric media was revived in 1983 by Gusev and Zheleznykh to be used for developing the radio-wave method of UHE $\left(>10^{14} \mathrm{eV}\right)$ cosmic neutrino detection [12]. It was suggested to build a large-scale neutrino detector (telescope) RAMAND using the ice in Antarctica as a neutrino target, taking advantage of very weak radio-wave absorption of ice at low temperatures at frequencies below a few $\mathrm{GHz}$ [12]. The proposed design involved deploying a surface grid of antennae listening to radio pulses in the frequency band from a few hundred $\mathrm{MHz}$ up to $1 \mathrm{GHz}$, produced by cascades from UHE neutrinos in the ice volume of $10^{9}-10^{11} \mathrm{~m}^{3}$. After experiments performed at the Soviet Antarctic Vostok station, the R\&D stage of RAMAND, an ice radio-wave detector with an effective volume up to $10 \mathrm{~km}^{3}$ was approved in 1988 by the Soviet authorities. A hexagonal form of the array with 1280 local stations placed on the $10 \mathrm{~km}^{2}$ detector area was chosen [13]. The main goal of the RAMAND project was to search for neutrinos with energies above a hundred $\mathrm{TeV}$ from astrophysical sources [14]. Unfortunately, RAMAND's work at Vostok was abruptly discontinued in 1991.

Some years later the Radio Ice Cherenkov Experiment (RICE [15]) with antennae placed at depths of 200-400 m in ice and the balloon-born Antarctic Impulsive Transient Antenna (ANITA [16]) experiments had been successfully conducted in Antarctica by D. Besson et al. and P. Gorham et al. Both experiments published limits on the UHE and EHE neutrino flux in the energy range $10^{16}-10^{21} \mathrm{eV}$. The constraints on the sensitivity of these experiments to the neutrino flux come from the relatively small effective volume of RICE, and relatively short exposure time, limited by the time of the balloon flight, in the case of ANITA.

An alternative design for an Antarctic UHE detection experiment that combines the strengths of RICE and ANITA, while having higher sensitivity and relatively low cost was proposed in [17]. In this paper, a possibility to place antennae on tethered balloons at a few kms above the ice surface was considered. The tethered scheme affords a large field of antenna view (compared to RICE), a high duty cycle (compared to ANITA), and can run in coincidence with a surface array (such as RAMAND).

Two huge radio neutrino detectors are currently being constructed as detectors for UHE and EHE astrophysical neutrinos based on the Cherenkov-Askaryan radio emission from neutrino-induced cascades of $10^{16}$ $10^{20} \mathrm{eV}$. The Askaryan Radio Array (ARA) [18] will be deployed in ice at the South Pole, covering $200 \mathrm{~km}^{2}$, with neutrino sensitivity of $10^{16}-10^{19} \mathrm{eV}$ to detect in particular GZK neutrinos. The Antarctic Ross Ice-Shelf Antenna Neutrino Array (ARIANNA) [19] (similar in concept to RAMAND) is a large surface antenna array.

In 1989 the Radio Astronomical Method of Hadron and Neutrino Detection (RAMHAND) project was proposed to use the Moon and even other celestial objects as giant targets for radio detectors of EHE hadrons and neutrinos [20]. The Moon had been considered as a perspective large-scale target and it was suggested to use large ground-based radio telescopes to search for Cherenkov-Askaryan radio pulses from cascades produced in the lunar regolith by EHE $\left(>10^{20} \mathrm{eV}\right)$ neutrinos and other cosmic particles. The possibility to detect radio emission of cascades of lower energies $\left(>10^{16} \mathrm{eV}\right)$ using a lunar satellite was also discussed.

\section{RAMHAND: The past and the prospects}

The first attempt to use a ground-based radio telescope as a detector and the lunar regolith as a target was undertaken 
by T.H. Hankins, R.D. Ekers and J.D. ÓSullivan in 1996 [21]. The observations were made at $1.5 \mathrm{GHz}$ frequency range using a 64-meter radio telescope of the Parks radio observatory (Australia). A 10-hour search for neutrino interacting with the lunar regolith had only put an upper limit to the isotropic EHE-neutrinos flux. The following attempts of much longer exposures on the Moon's limb undertaken by several teams around the world (see, for example, [22,23]) had put similar but much stronger restrictions to the flux of EHE neutrinos. For example, our 120-hour observations using the Kalyazin 64-m radio telescope (Russia) in the $1.4 \mathrm{GHz}$ and $2.3 \mathrm{GHz}$ frequency ranges [24] showed that the rate of the radio pulses resulting in interactions of neutrinos with energy above $10^{20} \mathrm{eV}$ with atoms of the lunar regolith does not exceed $(200 \mathrm{hrs})^{-1}$ or $1.410^{-6} \mathrm{sec}^{-1}$.

In subsequent years many more attempts were undertaken to register the bursts of Cherenkov radio emission from the Moon using the Westerbork synthesis radio telescopes (The Netherlands), some part of the VLA antennae (USA), again the Parks 64-m radio telescope, and other radio astronomy tools [25-29]. The new essentially stronger upper limit to the EHE neutrino flux was obtained from these experiments. These results were used to exclude some of the most exotic cosmological models.

At the same time a tendency could be mentioned in using lower frequencies for observations in these relatively recent RAMHAND-type experiments. New estimates of the optimal frequency band for such observations are based not only on the spectrum of the cascade's emission in the lunar regolith. They also take into account an increasing of the target volumes as well as less scattering of the cascade's emission at lower frequencies. Of course, an additional reason is the large dimensions of the existing low frequency radio telescopes. For example, the giant Low Frequency Array (LOFAR), constructed in Europe during the last decade [29] is now considered as a very high sensitive detector in RAMHAND-type experiments [30].

During the two last decades radio astronomers from Europe, Australia, South Africa and some other countries actively work on the SKA-project. First phases of this Square Kilometer Array SKA-LOW and SKA-MID are under construction now in Australia and in South Africa, respectively. Both multi-element radio telescopes should start working in the beginning of the 2020 s decade. Radio astronomers and HE-physicists hope to use both arrays as detectors of the Cherenkov-Askaryan radio pulses from the Moon [31,32]. Modelling of the cascades, their radio emission and corresponding detecting processes show that both arrays could be very effective tools not only for many kinds of classic radio astronomical tasks, but also for RAMHAND-type experiments.

Nevertheless, despite such promising prospects, astronomers are continuing their efforts to perfect the methods of detecting Cherenkov bursts from the Moon, and to apply them on the existing already radio telescopes. We are also working in this field, trying to improve our registration system and computer programs, which we use for on-line and off-line data reduction and analysis. First of all, we are going to analyze the data obtained during the monitoring of the large part of the sky with the Large Phased Array of the Pushchino Radio Astronomy Observatory (Fig. 1). This meridian radio telescope is one of the most sensitive meter wave length

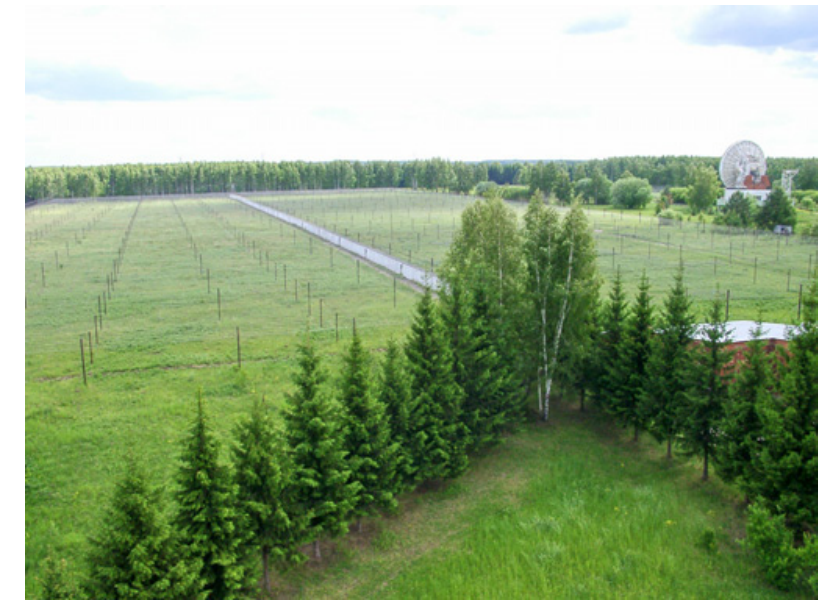

Figure 1. Large Phased Array of the Pushchino RAO ASC LPhI.

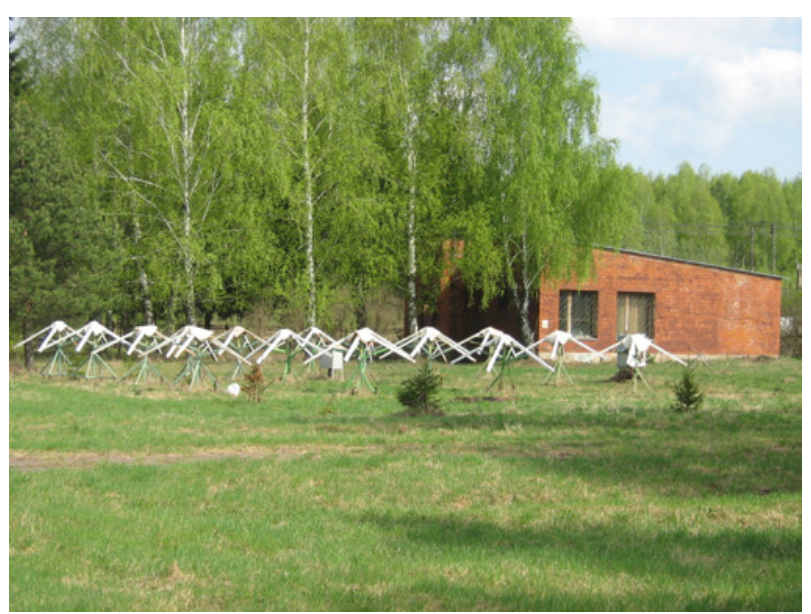

Figure 2. This 16 -element modulus is $1 / 8$ part of the future pathfinder in the Pushchino RAO.

radio telescopes. Its effective area is around $45000 \mathrm{~m}^{2}$ in t, he zenith (corresponding to a declination around $+55^{\circ}$ ) and decreases in the other directions as Cos Z. Beginning from mid-2013 this radio telescope has been used for day by day monitoring, at $111 \mathrm{MHz}$, of the large part of sky between declinations $\delta=-8^{\circ}$ and $+42^{\circ}$. We plan to carefully analyze all the tracks through the meridian plane by the Moon and hope to get around a 10-hour exposure using the data of the past three years. Multi-beam patterns of the phased array allow distinguishing various kinds of interference from the useful signal. We will try also to use another beam-forming system of the array and improve the time resolution of the data, but it will only be done for future observations.

Another possibility to get a new estimate of the extremely high energy cosmic rays and neutrinos, which we are planning to realize in the coming two or three years, is the implementation of monitoring the short radio bursts from the Moon at the small model of the meter-wave radio telescope with a wide field of view.

The model is constructed in Pushchino RAO (Fig. 2). It will be a wide-band phased array for the $40-65 \mathrm{MHz}$ frequency range and will have around $1000 \mathrm{~m}^{2}$ effective area. The field of view of this antenna will be around 2 steradians.

We plan to use it for monitoring different kinds of sporadic radio bursts as well as different transients and 
rather fast variations of the cosmic radio emission. The Moon will track through the radio telescope's field of view for 3 or 4 hours per day when its declination will be above $+15^{\circ}$. The model will consist of eight 16 -element modulus and will have 128-beam pattern. Certainly, we could not realize a good sensitivity with this model. It means that there will be a chance to only register particles above $10^{22} \mathrm{eV}$. At the same time we could hope to get with it a rather large exposure (around 300-350 hours per year). The registration system that will be worked out for this model could be later used for monitoring the Moon with other, much more sensitive, radio telescopes (for example, the Indian Ooty radio telescope).

\section{Simulations of cascades}

The new scheme for fast simulation of UHE cascades with energies $\mathrm{E}>10^{15} \mathrm{eV}$ and $\mathrm{EHE}$ cascades with energies up to $10^{20} \mathrm{eV}$ in dense media is suggested. At a later time this scheme will be used for calculations of radio emission of cascades with energies up to $10^{20} \mathrm{eV}$ and higher in the wide frequency band of $\sim 0.1-\mathrm{a}$ few $\mathrm{GHz}$.

Electron-photon cascades up to energies $10^{13}-10^{15} \mathrm{eV}$ may be simulated using GEANT-4 [33] with the standard cross sections of electromagnetic processes. Longitudinal and lateral charge excess profiles, track lengths of charged particles and other characteristics should be simulated. Results of these simulations should be approximated and stored. To reduce the CPU time for simulation of cascades at UHE energies a hybrid scheme was developed. This hybrid scheme had been suggested to estimate both the radio and acoustical signals caused by neutrinoinduced cascades with energies up to $10^{20} \mathrm{eV}$ in dense media (e.g. in ice, water and the lunar regolith). As a part of this hybrid scheme some original one-dimension Monte Carlo code was suggested to be used at higher energies. This original one-dimension Monte Carlo code will be exploited to simulate cascades up to $10^{20} \mathrm{eV}$ using approximations of results estimated with help of the GEANT4 package. This Monte Carlo code exploits the Migdal cross sections [34] to take into account the Landau-Pomeranchuck effect [35] now called the LandauPomeranchuck-Migdal (LPM) effect. This Monte Carlo code follows both electrons and gamma quanta up to some threshold energy $\mathrm{E}_{t h}$. The Monte Carlo approach should be initially used to take into account fluctuations in the development of the individual cascades. It should be mentioned that these fluctuations are very large at high energies because of the LPM effect. Due to this effect the mean interaction length may increase many times and become much larger than the cascade radiation length. For this reason, the cascade curve would have a stochastic form. A program which realizes this Monte Carlo approach for the Migdal cross sections for processes of pair creation and bremsstrahlung has been developed and tested. It should be mentioned that to calculate these cross sections accurately, very small energy bins (order of $10^{-4}$ ) have been used. It was found that the cascade length increases as $\mathrm{E}^{0.395}$ and at an energy of $10^{20} \mathrm{eV}$ it can exceed the value of $1 \mathrm{~km}$ (to be compared with the standard Bethe-Gaitler length of several dozen meters).

Below this threshold energy, $\mathrm{E}_{t h}$, approximated results of GEANT4 simulations are used to estimate the longitudinal and lateral charge excess profiles of charged

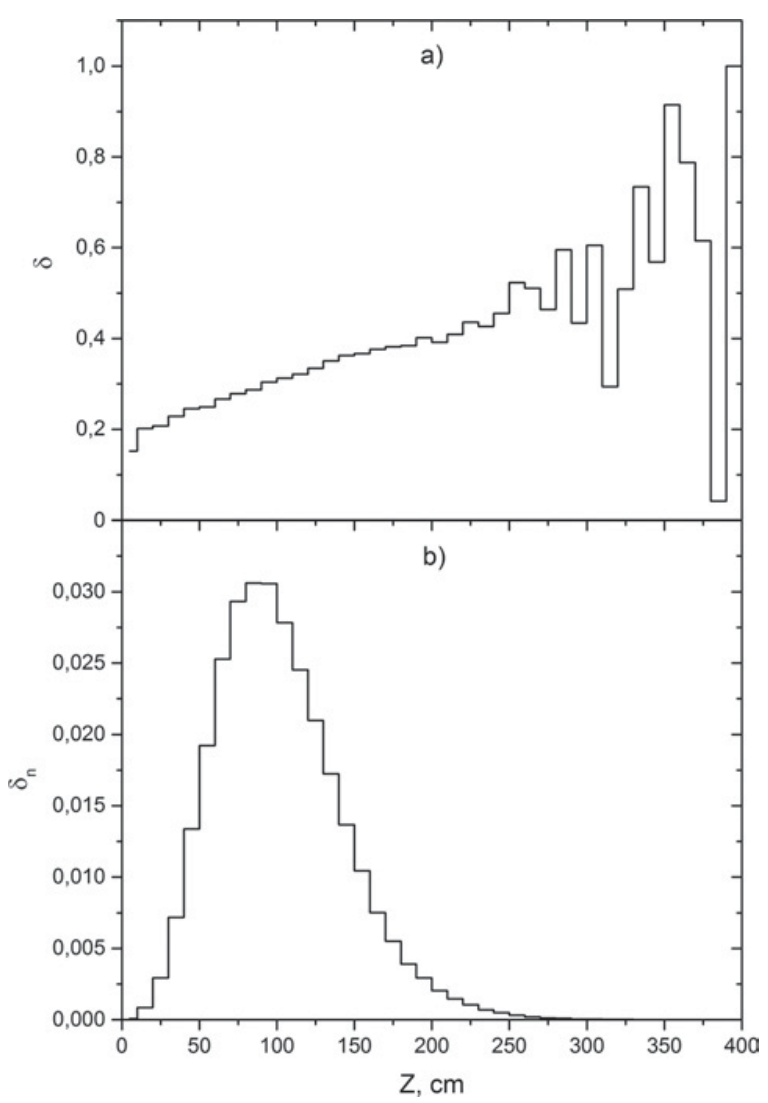

Figure 3. Longitudinal distribution of charge excess and its fraction.

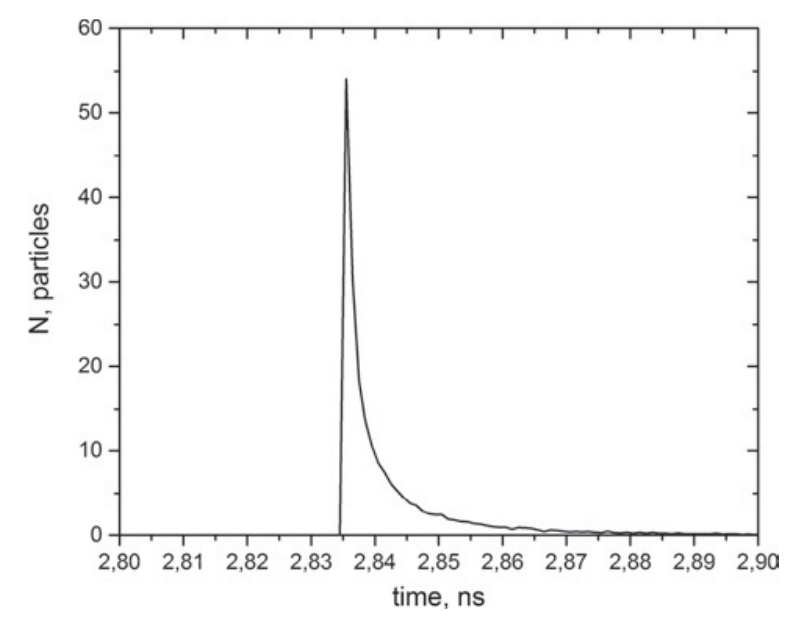

Figure 4. Time distribution of the shower front width.

particles in cascades induced by cosmic neutrinos. Then the radio emission of such cascades may be calculated. Some profiles for the total number of particles (electrons + positrons) as well as the charge excess (electrons - positrons) in showers initiated in the lunar regolith $\left(\mathrm{d}=1.41 \mathrm{~g} / \mathrm{cm}^{3}\right)$ by gamma quanta with various energies have been calculated using a kinetic energy threshold of $0.1 \mathrm{MeV}$. Some results are shown in Figs. 3-5. Figure 3 shows a longitudinal distribution for both the charge excess $\delta(z)$ and its relative fraction $\delta n(z)$ for a gamma shower with energy $\mathrm{E}=100 \mathrm{GeV}$. It is seen that the charge excess at the maximum of the shower $\mathrm{z}=100 \mathrm{~cm}$ is slightly below $30 \%$.

The time distribution of particles which are crossing the position of shower maximum is shown in Fig. 4 for 


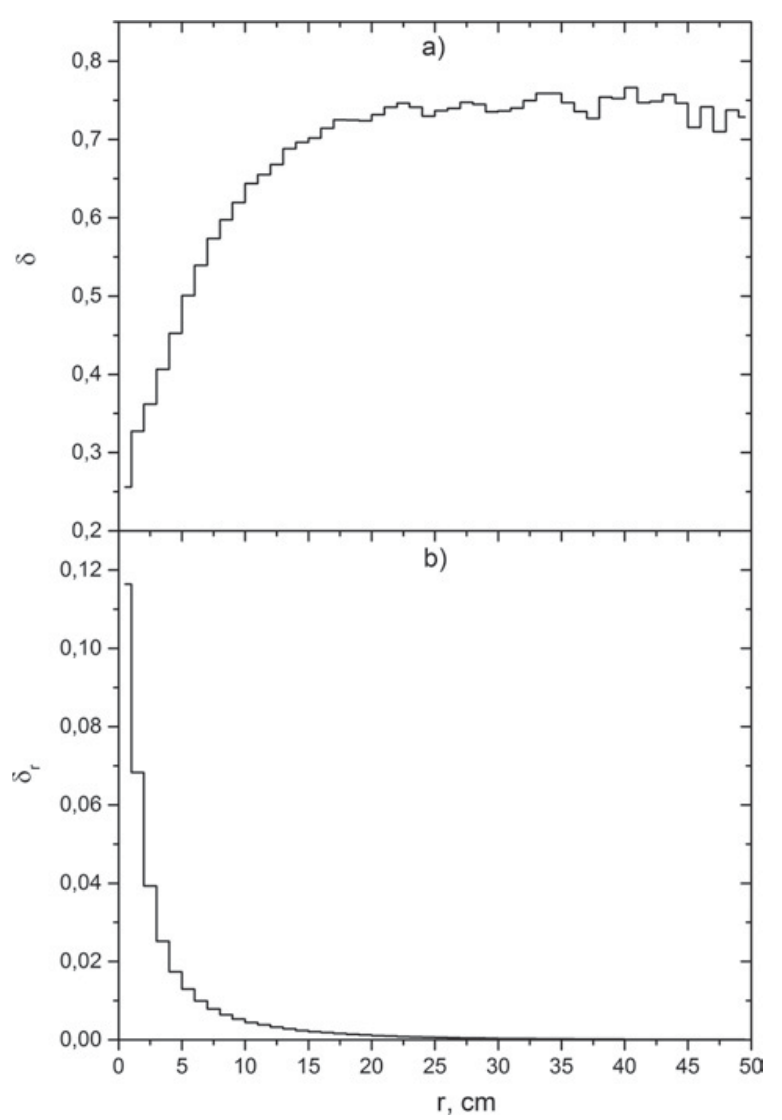

Figure 5. Lateral distribution of charge excess and its fraction.

a gamma shower with energy $\mathrm{E}=100 \mathrm{GeV}$. It is seen that the total width of this distribution is slightly below $0.04 \mathrm{~ns}$ which corresponds to nearly $\sim 1 \mathrm{~cm}$ width of the shower front.

The radial distribution of the charge excess, $\delta(r)$, and its relative fraction, $\delta \mathrm{n}(\mathrm{r})$, are shown in Fig. 5 for a gamma shower with energy $\mathrm{E}=100 \mathrm{GeV}$. It is seen that the charge excess at $\mathrm{r} \sim 1 \mathrm{~cm}$ is nearly equal to $30 \%$. So, it is possible to conclude that both the time and radial distributions show widths of the shower charge excess of the order of $1-5 \mathrm{~cm}$. So, detection of radio emission with frequencies $(v=c / \lambda)$ below $3 \mathrm{GHz}$ is possible. The charge excess distribution displays $25-30 \%$ negative excess near the maximum position.

\section{Conclusion}

At the beginning of the 2000 s V.L. Ginsburg wrote in his book "About Science, Myself and Others": "At last, it is literally on an exit there is Neutrino Astronomy of High Energies $>10^{12} \mathrm{eV}$ '[36]. And the first astrophysical HE neutrinos had been registered by a team of the gigaton optical HE neutrino telescope IceCube in Antarctica in 2013. It happened as a result of the very successful development of the optical method of deep under-ice neutrino detection during 25 years.

The development of multi gigaton radio detectors for UHE $\left(>10^{15}\right)$ and EHE $\left(>10^{18}\right)$ Neutrino Astronomy, using the Antarctic ice (RAMAND, 1983-1991) and the lunar regolith (RAMHAND, since 1989), had started in the framework of the Russian (Soviet) DUMAND program more than 30 years ago.
It is possible to now say that it is literally on an exit there is UHE and EHE Neutrino Astronomy: the new generation of huge radio neutrino telescopes under construction in Antarctica ARA and ARIANNA are designed as discovery instruments for astrophysical neutrinos of $\mathrm{E}>10^{16}-10^{17} \mathrm{eV}$.

Using the new larger-scale radio astronomical telescopes (SKA and others) for lunar experiments would give great possibilities for studying fundamental problems of Cosmic Ray (Astroparticle) Physics and Neutrino Astronomy in the interval of energies more than $\mathbf{1 0}^{\mathbf{2 0}} \mathbf{e V}$.

This work was supported by RFBR (grant 16-29-130740). The authors are deeply indebted to Bryan Pattison for valuable discussions.

\section{References}

[1] M.A. Markov, Proc. 10th Int. Conf. on High-Energy Physics at Rochester, 579 (1960)

[2] I.M. Zheleznykh, Thesis, Moscow State University, Physical Faculty (1958)

[3] M.A. Markov, I.M. Zheleznykh, Nucl. Phys. 27, 385 (1961)

[4] F. Reines, J. Sellschop, Scientific American, February (1966)

[5] A. Roberts, Rev. Mod. Phys. 64, 259 (1992)

[6] J. Learned, Ch. Spiering, CERN Courier, July/August (2016)

[7] F. Halzen, J. Learned, "High energy neutrino detection in deep polar ice", preprint U. Wisconsin, June (1988)

[8] M.A. Markov, I.M. Zheleznykh, Proc. 1979 DUMAND Workshop at Khabarovsk and lake Baikal, ed. J. Learned, Honolulu, 177 (1980)

[9] L.G. Dedenko, M.A. Markov, I.M. Zheleznykh, Proc. Neutrino-1981, vol. 1, eds. R Cence, E. Ma and A. Roberts, Maui, Hawaii, 292 (1981)

[10] M.A. Markov, I.M. Zheleznykh, Nucl. Instr. Meth. A 248, 242 (1986)

[11] G.A. Askaryan, Sov. Phys. JETP 14, 441 (1962)

[12] G.A. Gusev, I.M. Zheleznykh, JETP Letters 38, 505 (1983)

[13] A.L. Provorov et al., Proc. 3rd Int. Workshop on Neutrino Telescopes, Venice, ed. Milla Baldo-Ceolin, 337 (1991)

[14] A.L. Provorov, I.M. Zheleznykh, Astroparticle Physics 4, 55 (1995)

[15] I. Kravchenko, et al., Physical Review D 73, 082002 (2006)

[16] P. Gorham, et al., Physical Review Letters 103, 051103 (2009)

[17] D. Besson, R. Dagkesamanskii, I Kravchenko, et al., Nuclear Instr. \& Methods in Phys. Res. A 662, 50 (2012)

[18] P. Alison, et al., Astroparticle Physics 70, 62 (2015)

[19] S.W. Barwick, et al., Astroparticle Physics 12, 62 (2015)

[20] R.D. Dagkesamanskii, I.M. Zheleznykh, Sov. Phys. JETP 50, 259 (1989)

[21] T.H. Henkins, R.D. Ekers, J.D. OŚullivan, Monthly Notices of the Roy. Astron. Soc. 283, 1027 (1996)

[22] P.W. Gorham, C.L Hebert, K.M. Liewer, et al., Phys. Rev. Lett. 93, 041101 (2004) 
[23] A.R. Beresnyak, R.D. Dagkesamanskii, I.M. Zheleznykh, et al., Astron. Rep. 49, 127 (2005)

[24] C.W. James, R.D. Ekers, J. Alvarez-Muniz, et al., Phys. Rev. D 81, 042003 (2010)

[25] R.E. Spencer, A. Macfarlane, O. Mills, L. Piccirillo, Proc. 10th EVN Symposium, 097 (2010)

[26] T.R. Jaeger, R.L. Mutel, K.G. Gayley, Astropart. Phys. 43, 293 (2010)

[27] S. Buitink, O. Scholten, J. Bacelar, et al., Astron. \& Astrophys. 521, A47 (2010)

[28] J.D. Bray, R.D. Ekers, C.W. James, et al., Proc. 32nd ICRC (2011)

[29] N.P. van Haarlem , M.W. Wise, A.W. Gunst, et al., LOFAR: The Low Frequency Array, arXiv: 1305.3550

[30] K. Singh, M. Mevius, O. Scholten, et al. (LOFAR Coll.), Nuclear Instr. \& Methods in Phys. Res. A 664, 171 (2012)
[31] J.D. Bray, J. Alvarez-Muniz, S. Buitink, R.D. Dagkesamanskii, R.D. Ekers, et al., "Lunar detection of ultra-high-energy cosmic rays and neutrinos with the Square Kilometre Array", Proceedings of Science, AASKA14, 144, in Advancing of Astrophysics with Square Kilometer Array, 1, 961 (2015). See also arXiv: 1408.6069v2 [astro-ph. IM] 19, Dec. 2014

[32] C. James, on behalf of the SKA High Energy Cosmic Particles (lunar) Focus Group, Proceedings of the ARENA 2016, Groningen, The Netherlands

[33] Geant4 User's Guide for Toolkit Developers http://geant4.web.cern.ch/geant4/support/ userdocuments

[34] A.B. Migdal, Phys. Rev. 103(6), 1811 (1956)

[35] L.D. Landau, I.Ya. Pomeranchuk, Dokladi AN USSR 92, 535 (1953)

[36] V.L. Ginzburg, "About science, myself and others", Moscow, Fizmatlit (2003) 